\title{
Shift of Glucose Peak Time During Oral Glucose Tolerance Test is Associated with Changes in Insulin Secretion and Insulin Sensitivity After Therapy with Antidiabetic Drugs in Patients with Type 2 Diabetes
}

Yanqiu Jiang · Shiwei Cui · Rongping Zhang • Xiaoqin Zhao $\cdot$ Lili Yao $\cdot$ Rong OuYang $\cdot$ Wei Chen $\cdot$ Ranran Zhou Xuying Zhao · Zhuqi Tang · Jin Yuan · Jie Yuan · Chen Qian · Ping Huang • Yunjuan Gu (D) · Xinlei Wang (D)

Received: May 28, 2021 / Accepted: June 23, 2021 / Published online: August 3, 2021

(C) The Author(s) 2021

\section{ABSTRACT}

Introduction: Delay in peak blood glucose during an oral glucose tolerance test (OGTT) predicts declining $\beta$-cell function and poor ability to regulate glucose metabolism. Glucose peak time has not been used as a comparative indicator of the improvement in islet function after treatment with exenatide, insulin, or oral antidiabetic drugs (OADs). We evaluated the efficacy of three types of antidiabetic drugs on the basis of blood glucose peak time in patients with non-newly diagnosed type 2 diabetes.

Methods: The data from 100 patients with diabetes who completed two OGTTs within 6 months were collected. Thirty-seven of them with type 2 diabetes were treated with Humalog

Yanqiu Jiang and Shiwei Cui contributed equally to this work and are joint first authors.

Y. Jiang · S. Cui - R. Zhang $\cdot$ X. Zhao $\cdot$ L. Yao

R. OuYang - W. Chen - R. Zhou - X. Zhao ·

Z. Tang $\cdot$ J. Yuan $\cdot$ J. Yuan $\cdot$ P. Huang ·

Y. Gu $(\bowtie) \cdot X$. Wang $(\bowtie)$

Department of Endocrinology and Metabolism,

Affiliated Hospital of Nantong University, 20 Xi-si

Road, Nantong 226001, Jiangsu, China

e-mail: desettle@ntu.edu.cnX. Wang

e-mail: rubi221@126.com

C. Qian

Center of Laboratory Medicine, Affiliated Hospital of Nantong University, Nantong 226001, Jiangsu, China
Mix25, 28 patients with OADs (metformin, acarbose, and gliclazide), and 35 patients with exenatide.

Results: Glycated hemoglobin improved in all three groups after treatment $(P<0.05)$. Subcutaneous adipose tissue $(P<0.01)$ and visceral adipose tissue $(P<0.0001)$ significantly decreased in the exenatide group. The insulinogenic index (IGI) $(P=0.01)$ and IGI $\times$ oral glucose insulin sensitivity (OGIS) $(P=0.01)$ improved in the exenatide group only. Homeostatic assessment of $\beta$-cell function (HOMA- $\beta$ ) and OGIS were greater in the exenatide and OAD groups than in the Humalog Mix25 group (all $P<0.05$ ). A shift to an earlier peak was observed in $57.1 \%, 35.7 \%$, and $27.0 \%$ of patients in the exenatide, OAD, and Huma$\log$ Mix25 groups, respectively $(P=0.029)$. OGIS (odds ratio [OR] 0.54, 95\% confidence interval $[\mathrm{CI}] 0.33-0.89, P=0.026)$ and IGI $\times$ OGIS (OR 1.72, 95\% CI 0.44-6.68, $P=0.012$ ) were independently related to shifts in glucose peak time.

Conclusion: Exenatide, Humalog Mix25, and OADs improved glycemic metabolism. However, exenatide exhibited superior efficacy in shifting blood glucose peak time to an earlier point, while it improved insulin secretion and insulin sensitivity. Hence, the shift of glucose peak time may be considered an indicator for the evaluation of the effect of hypoglycemic drugs. 
Keywords: Exenatide; Glucose peak time; Hypoglycemic agents; Oral glucose tolerance test; Type 2 diabetes

\section{Key Summary Points}

Blood glucose peak time was used to evaluate the efficacy of three types of antidiabetic drugs in patients with nonnewly diagnosed type 2 diabetes.

The insulinogenic index (IGI) and IGI $\times$ oral glucose insulin sensitivity (OGIS) improved in the exenatide group.

A shift to an earlier peak was observed in $57.1 \%, 35.7 \%$, and $27.0 \%$ of patients in the exenatide, oral antidiabetic drugs (OAD), and Humalog Mix25 groups, respectively.

Exenatide exhibited superior efficacy in shifting blood glucose peak time to an earlier point, while it improved insulin secretion and insulin sensitivity.

\section{INTRODUCTION}

Insufficient insulin secretion and insensitivity to insulin are the main characteristics of type 2 diabetes mellitus (T2DM). The current gold standard method for evaluating insulin secretion and sensitivity is the hyperinsulinemic euglycemic glucose clamp test, which maintains the plasma exogenous insulin at a high level and glucose at a basal steady-state level [1]. However, this method is invasive, complex, and expensive; therefore, its application in clinical practice is limited. To facilitate the evaluation of insulin secretion and sensitivity, researchers have proposed more practical alternative indicators. Surrogate indices are extensively used to evaluate insulin sensitivity and pancreatic $\beta$-cell function. For example, oral glucose tolerance test (OGTT)-derived indicators have become risk indicators of early changes in $\beta$-cell function in different populations [2]. These indices include oral deposition, Raynaud, Cederholm, fasting
Belfiore, ISI, QUICKI, Matsuda indexes, etc. [3]. However, OGTT-derived indices require complex calculations; therefore, their applications in clinical practice are limited. In addition, OGTTderived indices deviate from actual clinical results; for example, homeostatic assessment of insulin resistance (HOMA-IR) was presumed to have equivalent insulin sensitivity in the liver, muscle, and adipose tissues, but these parameters actually differ in distinct tissues [4]. Moreover, the accuracy of HOMA-IR and HOMA- $\beta$ may be limited in fasting hyperglycemia [5]. Research on the relationship between OGTT-derived indices and the clamp test has demonstrated that the specificity and sensitivity of insulin secretion and resistance assessed using OGTT-derived indices are poorer than those of the hyperinsulinemic euglycemic glucose clamp test [6].

To the best of our knowledge, different blood glucose curve phenotypes in OGTT represent different states of islet function [7]. Recently, the complex curve shape has been considered to indicate better islet function in adults [8]. However, there is heterogeneity in the shape of the glucose curve, indicating poor reproducibility [9]. Therefore, researchers have been trying to devise convenient, novel, and reproducible parameters of insulin and glucose response to OGTT to evaluate a patient's islet function.

Time to glucose peak is a fresh evaluation index on the OGTT. The peak blood glucose time during OGTT is an important tool that can strengthen the risk stratification of prediabetes and can be used to assess the risk of T2DM $[10,11]$. Furthermore, compared with the blood glucose curve shape, the glucose peak time displayed reliable reproducibility on replicate testing $(k=0.76)$ [9]. Another advantage is that the delay time of peak blood glucose is related to pancreatic $\beta$-cell dysfunction [12]. Moreover, subsequent studies have demonstrated that the insulin sensitivity and secretion of patients with T2DM can be reflected by the peak blood glucose time [10]. Tran et al. used glucose peak time as an indicator to compare the efficacy of liraglutide treatment in patients with earlyphase T2DM, confirming that this indicator was associated with insulin sensitivity and secretion [12]. However, for patients with long-term T2DM, the effect of different antidiabetic drugs 
on the peak blood glucose time remains unknown. D'Alessio et al. reported that a 24-week exenatide treatment can improve the islet function of patients with early T2DM. On the basis of this finding, we set the treatment cycle to 6 months [13]. Previous studies have reported that insulin, metformin, acarbose, and sulfonylureas can also protect pancreatic $\beta$-cells $[14,15]$. Meanwhile, a delay of the peak blood glucose time indicates a decline of the patient's islet function. Thus far, the glucose peak time has not been used as a comparative indicator to evaluate the improvement in islet function after treatment with exenatide, insulin, or oral antidiabetic drugs (OAD; metformin, acarbose, and gliclazide).

This study aimed to evaluate the variabilities in insulin secretion and sensitivity, metabolic characteristics, and ectopic fat accumulation in patients with diabetes after therapy with exenatide, insulin, or OADs. Simultaneously, we validated whether the peak time of glucose can be used to evaluate the efficacy of antidiabetic treatments in individuals with non-newly diagnosed T2DM.

\section{METHODS}

\section{Study Design and Participants}

We retrospectively analyzed the data from patients who underwent the 3-h OGTT in the Endocrinology Department of Nantong University Hospital, Nantong, China, between February 2015 and June 2018. The inclusion criteria were as follows: (i) diagnosed with T2DM according to American Diabetes Association standards; (ii) age over 18 years old; (iii) prescribed exenatide, or Humalog Mix25, or OADs (metformin, acarbose, and sulfonylureas); (iv) underwent two complete OGTTs, one at the beginning and the other 6 months after therapy; (v) used a stable dose of OAD for at least 3 months (did not use glucagon-like peptide 1 receptor agonists (GLP-1RAs) or insulin) before the first OGTT. Patients who met any of the following conditions were excluded: (i) impaired liver or kidney function, malignant tumors, and active infection; (ii) pregnancy or breastfeeding; (iii) acute diabetic complications.
Exenatide was subcutaneously injected within $60 \mathrm{~min}$ before breakfast and dinner (or before two main meals a day with a dosing interval of $6 \mathrm{~h}$ or longer). Humalog Mix25 (75\% neutral protamine lispro, $25 \%$ lispro) was injected subcutaneously twice a day. OADs included metformin, acarbose, and gliclazide.

This retrospective study, which collected patient clinical data, did not interfere with the treatment plan of the patients. The researchers will protect the security of personal information provided by the patient. All participants signed an informed consent form in the study. The protocol was approved by the Ethics Committee of the Affiliated Hospital of Nantong University (approval number 2015-K002-D01). The study was registered with Chinese Clinical Trial Registry (ChiCTR-IPR-14005568). The study was carried out in line with the Helsinki Declaration of 1964 and its later amendments.

\section{Lifestyle Intervention}

All patients received diabetes education including guidance of diet and exercise at our endocrinology clinic by specialist doctors and nurses before receiving the first OGTT.

\section{Anthropometric Measurements}

Patients' body weight and height were measured by nurses. Weight was measured in kilograms, with patients wearing the lightest clothing. Height was recorded in centimeters, with the patients standing barefoot. Data were recorded using a height- and body weightmeasuring instrument (Tanita TBF-300, Japan). Electronic sphygmomanometers (Omron HEM6000 , Osaka, Japan) were used to measure systolic blood pressure (SBP) and diastolic blood pressure (DBP). Waist circumference was determined using a soft ruler to measure the horizontal circumference of the thinnest part of the waist at the end of exhalation and before the start of inhalation. Body mass index (BMI) was calculated according to the following formula: BMI = body weight $(\mathrm{kg}) /[\text { height }(\mathrm{m})]^{2}$. Visceral adipose tissue (VAT) and subcutaneous adipose tissue (SAT) were obtained by MRI (1.5-T MRI 
system, Milwaukee, USA) scanning from the 12 th thoracic vertebra to the 1 st sacral vertebra through breathing gating technology.

\section{OGTT, Insulin, and C-Peptide Release Tests}

The patients underwent two 3-h OGTTs and ingested $75 \mathrm{~g}$ of glucose each time. Before the two OGTT implementations, all patients stopped taking hypoglycemic drugs for 3 days. Glucose, insulin, and C-peptide levels were determined using venous blood specimens acquired at $0,30,60,90,120,150$, and $180 \mathrm{~min}$. Plasma glucose was examined using a standard laboratory procedure (Siemens ADVIA ${ }^{\circledR}$ 2400). Insulin levels and C-peptide concentrations were determined using chemiluminescent methods (Cobas e411; Roche, Switzerland).

\section{Biochemical Measurements}

Triglycerides (TG), glycated hemoglobin (HbA1c), and total cholesterol (TC) levels were determined using fasting blood samples (at least $8 \mathrm{~h}$ of fasting). High-performance liquid chromatography (VARIANT ${ }^{\mathrm{TM}}$ II, Hercules, USA) was used to determine HbA1c. Blood lipid parameters were measured using enzymatic methods (ADVIA ®2400; Siemens, Germany).

\section{Calculation of Variables}

Islet secretion function was defined using two methods: (1) HOMA- $\beta \quad[16]=(20 \times$ fasting insulin (FINS) $[\mathrm{mIU} / \mathrm{L}]) /($ fasting glucose $(\mathrm{FG})$ $[\mathrm{mmol} / \mathrm{L}]-3.5)$ or (2) insulinogenic index (IGI) $[17]=\Delta \mathrm{I} 0-30 \mathrm{~min} / \Delta \mathrm{GO}-30 \mathrm{~min}$. Insulin sensitivity was evaluated using two methods: (1) oral glucose insulin sensitivity (OGIS) [5] (http:// webmet.pd.cnr.it/ogis/ index.php) or (2) HOMA-IR [5] $=$ FG $(\mathrm{mmol} / \mathrm{L}) \times$ FINS $(\mathrm{mIU} / \mathrm{L}) /$ 22.5. Disposition indices $=$ IGI $\times$ OGIS or HOMA-IS [6] $\times$ HOMA- $\beta$, whereby HOMA-IS = 1/HOMA-IR. The area under the curve (AUC) of serum glucose $\left(\mathrm{AUC}_{\mathrm{GO}-180 \mathrm{~min}}\right)$, insulin (AUC ${ }_{\text {INSO-180min }}$ ), and C-peptide (AUC $\mathrm{C}_{\text {-peptideo- }}$ 180min) through OGTT was calculated using trapezoidal rules.

\section{Definition of Shifts in Blood Glucose Peak Time}

The peak blood glucose time was defined as the highest point of blood glucose among the seven points during OGTT. The change in peak blood glucose time was based on the relationship between the baseline data and the 6-month period data during OGTTs. On the basis of these changes, we divided patients into three categories: shifted to an earlier, unchanged, or a later time point.

\section{Statistical Analyses}

Normally distributed data were expressed as mean \pm standard deviation. Non-normally distributed variables were logarithmically transformed before analysis or presented as median (25th-75th percentiles). The relationships between hypoglycemic drugs and variables such as age, diabetes duration, BMI, waist circumference, SBP, DBP, fasting blood glucose (FBG), VAT, SAT, TC, TG, HbA1c, HOMA- $\beta$, and HOMA-IR, among others, were analyzed by oneway analysis of variance or Mann-Whitney $U$ test. The chi-square test was used to compare groups with regard to shifts in peak glucose time according to the number of people. Risk factor analysis was performed using multinominal logistic regression analysis. SPSS, version 25.0 (IBM Corp., Armonk, NY, USA), was used for all statistical analyses. Statistical significance was placed at $P<0.05$.

\section{RESULTS}

\section{Clinical Characteristics}

The data from 100 patients were analyzed. Thirty-seven patients were treated with Huma$\log$ Mix 25, 28 patients with oral hypoglycemic drugs, and 35 patients with exenatide. The clinical characteristics of the participants are detailed in Table 1 . No significant differences were observed in the diabetes duration, age, height, body weight, BMI, DBP, waist circumference, FBG, 2hPBG, HbA1c, TC, and TG 
Table 1 Baseline characteristics of group exenatide, Humalog Mix25, and oral antidiabetic drugs

\begin{tabular}{|c|c|c|c|c|}
\hline & Pre-exenatide $(n=35)$ & Pre-Humalog Mix25 $(n=37)$ & Pre-OADs $(n=28)$ & $P$ value \\
\hline Age (years) & $55.78 \pm 7.08$ & $57.62 \pm 11.33$ & $55.57 \pm 9.69$ & 0.67 \\
\hline Disease duration (years) & $8.00(1.00,12.00)$ & $9.00(4.00,12.00)$ & $8.00(3.00,10.00)$ & 0.58 \\
\hline Height $(\mathrm{cm})$ & $166.94 \pm 9.41$ & $165.00 \pm 6.66$ & $166.02 \pm 7.27$ & 0.63 \\
\hline Weight (kg) & $66.85 \pm 8.96$ & $64.47 \pm 6.63$ & $65.53 \pm 6.72$ & 0.47 \\
\hline $\operatorname{BMI}\left(\mathrm{kg} / \mathrm{m}^{2}\right)$ & $24.00 \pm 0.30$ & $23.30 \pm 0.24$ & $23.56 \pm 0.31$ & 0.69 \\
\hline Waistline $(\mathrm{cm})$ & $89.18 \pm 1.69$ & $87.66 \pm 0.87$ & $88.88 \pm 1.28$ & 0.79 \\
\hline SBP $(\mathrm{mmHg})$ & $123.18 \pm 4.35$ & $134.41 \pm 3.40$ & $131.08 \pm 4.53$ & 0.03 \\
\hline $\mathrm{DBP}(\mathrm{mmHg})$ & $77.00(70.00,84.00)$ & $73.00(70.00,85.00)$ & $78.00(75.00,80.00)$ & 0.89 \\
\hline $\mathrm{FBG}(\mathrm{mmol} / \mathrm{L})$ & $8.32 \pm 2.09$ & $9.07 \pm 2.22$ & $8.06 \pm 1.83$ & 0.74 \\
\hline $2 \mathrm{hPBG}(\mathrm{mmol} / \mathrm{L})$ & $12.57 \pm 3.72$ & $14.96 \pm 3.82$ & $13.53 \pm 3.41$ & 0.12 \\
\hline HbAlc (\%) & $8.80(8.03,9.30)$ & $8.20(7.40,8.95)$ & $7.30(7.15,9.25)$ & 0.26 \\
\hline $\mathrm{TC}(\mathrm{mmol} / \mathrm{L})$ & $5.30 \pm 0.22$ & $4.99 \pm 0.15$ & $4.74 \pm 0.22$ & 0.15 \\
\hline TG $(\mathrm{mmol} / \mathrm{L})$ & $0.36 \pm 0.30$ & $0.23 \pm 0.15$ & $0.24 \pm 0.18$ & 0.82 \\
\hline
\end{tabular}

TG was logarithmically transformed. Data are expressed as the mean \pm standard deviation or median (interquartile range) $B M I$ body mass index, $S B P$ systolic blood pressure, $D B P$ diastolic blood pressure, $F B G$ fasting blood glucose, $2 h P B G 2$-h postprandial blood glucose, $H b A 1 c$ glycated hemoglobin, $T C$ total cholesterol, $T G$ triglycerides

among groups at baseline. The Humalog Mix25 group had higher SBP than the other groups $(P=0.03)$. After 6 months of treatment, glycated hemoglobin was improved in all three groups $(P<0.05)$. Body weight, BMI, and waistline decreased in the exenatide and $\mathrm{OAD}$ groups (all $P<0.05$ ). FBG and 2hPBG levels were decreased in the exenatide $(8.32 \pm$ $2.09 \mathrm{mmol} / \mathrm{L}$ vs. $6.91 \pm 1.68 \mathrm{mmol} / \mathrm{L}, P=0.68$; $12.57 \pm 3.72 \mathrm{mmol} / \mathrm{L}$ vs. $10.81 \pm 3.38 \mathrm{mmol} / \mathrm{L}$, $P=0.13)$, Humalog Mix25 $(9.07 \pm 2.22 \mathrm{mmol} /$ $\mathrm{L}$ vs. $7.11 \pm 1.50 \mathrm{mmol} / \mathrm{L}, P=0.026 ; 14.96 \pm$ $3.82 \mathrm{mmol} / \mathrm{L}$ vs. $\quad 12.64 \pm 3.09 \mathrm{mmol} / \mathrm{L}, \quad P=$ $0.64)$, and $\mathrm{OAD}(8.06 \pm 1.83 \mathrm{mmol} / \mathrm{L}$ vs. 7.05 $\pm 2.13 \mathrm{mmol} / \mathrm{L}, P=0.48 ; 13.53 \pm 3.41 \mathrm{mmol} / \mathrm{L}$ vs. $\quad 11.59 \pm 4.44 \mathrm{mmol} / \mathrm{L}, \quad P=0.23$ ) groups. Only differences in FBG in the Humalog Mix25 group pre- and post-treatments were significant. SAT and VAT were decreased in the exenatide group $\left(122.21 \pm 8.99 \mathrm{~cm}^{2}\right.$ vs. $104.11 \pm 8.84$ $\mathrm{cm}^{2}, P<0.01 ; 77.96 \pm 7.03 \mathrm{~cm}^{2}$ vs. $60.28 \pm$ $\left.6.19 \mathrm{~cm}^{2}, \quad P<0.0001\right)$. Adipose deposition increased after Humalog Mix25 treatment and decreased after OAD therapy, albeit without any statistically significant difference. TC $(5.30 \pm 0.22 \mathrm{mmol} / \mathrm{L}$ vs. $4.90 \pm 0.29 \mathrm{mmol} / \mathrm{L}$, $P<0.00001)$ and TG $(2.24 \pm 0.65 \mathrm{mmol} / \mathrm{L}$ vs. $1.19 \pm 0.24 \mathrm{mmol} / \mathrm{L}, \quad P=0.03)$ levels significantly decreased in the exenatide group (Table 2).

\section{Glucose Tolerance, Insulin, and C-Peptide Variation}

Glucose peaks of all groups occurred at $120 \mathrm{~min}$ after baseline (Fig. 1a). After 6 months of treatment, the glucose peaks of the exenatide and OAD groups occurred at $90 \mathrm{~min}$, while that of the Humalog Mix25 group remained at $120 \mathrm{~min}$

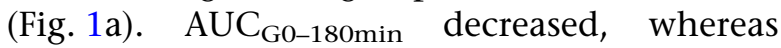
AUC ${ }_{\text {INSO-180min }}$ and AUC $_{\text {C-peptide0-180min }}$ increased in all groups after the 6-month therapy. In particular, $\mathrm{AUC}_{\mathrm{INSO}-180 \mathrm{~min}}(P=0.02)$ and AUC $_{\mathrm{C} \text {-peptide0-180min }}(P=0.003)$ in the exenatide group were statistically significant after 6 months of treatment (Table 2). 


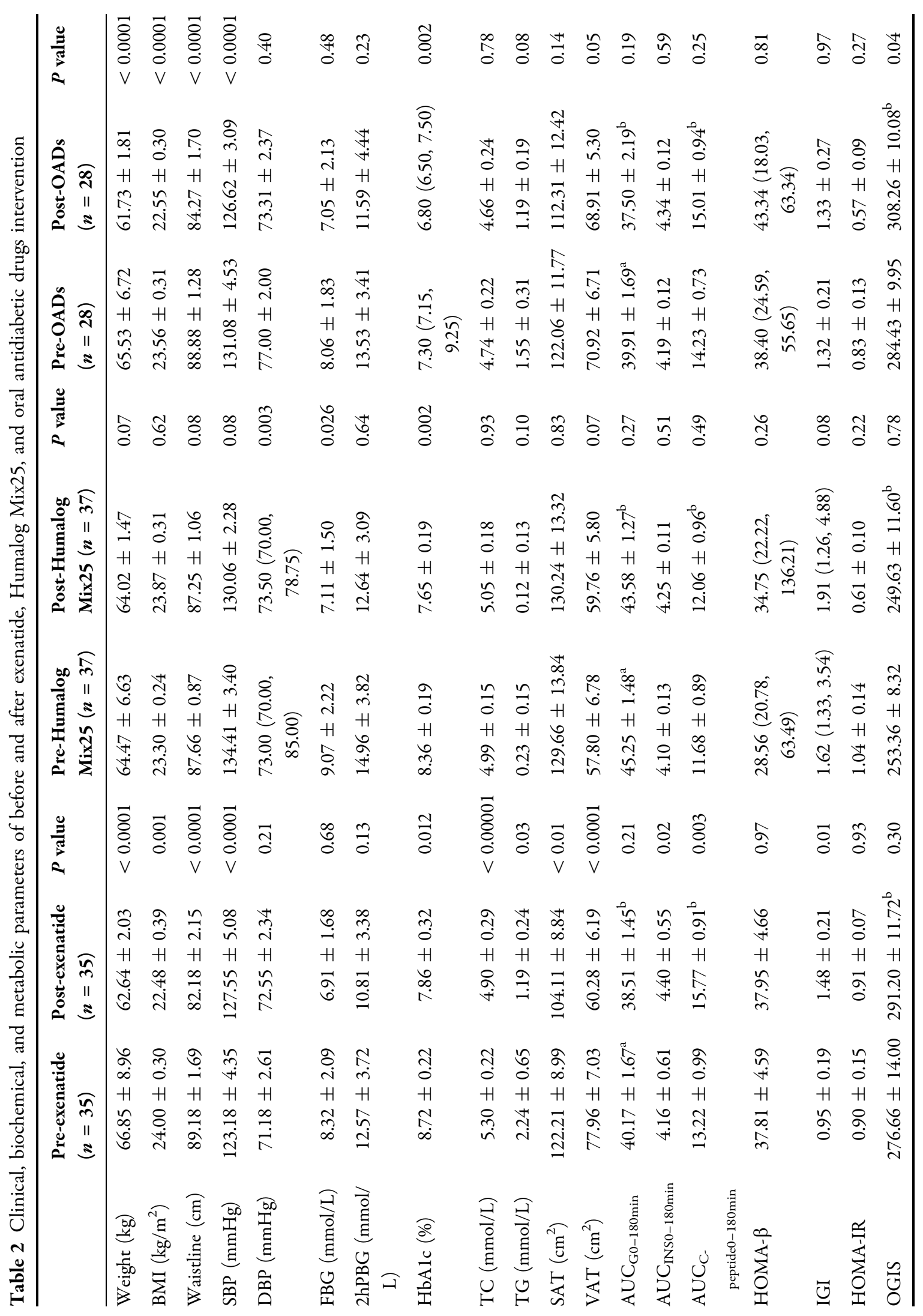




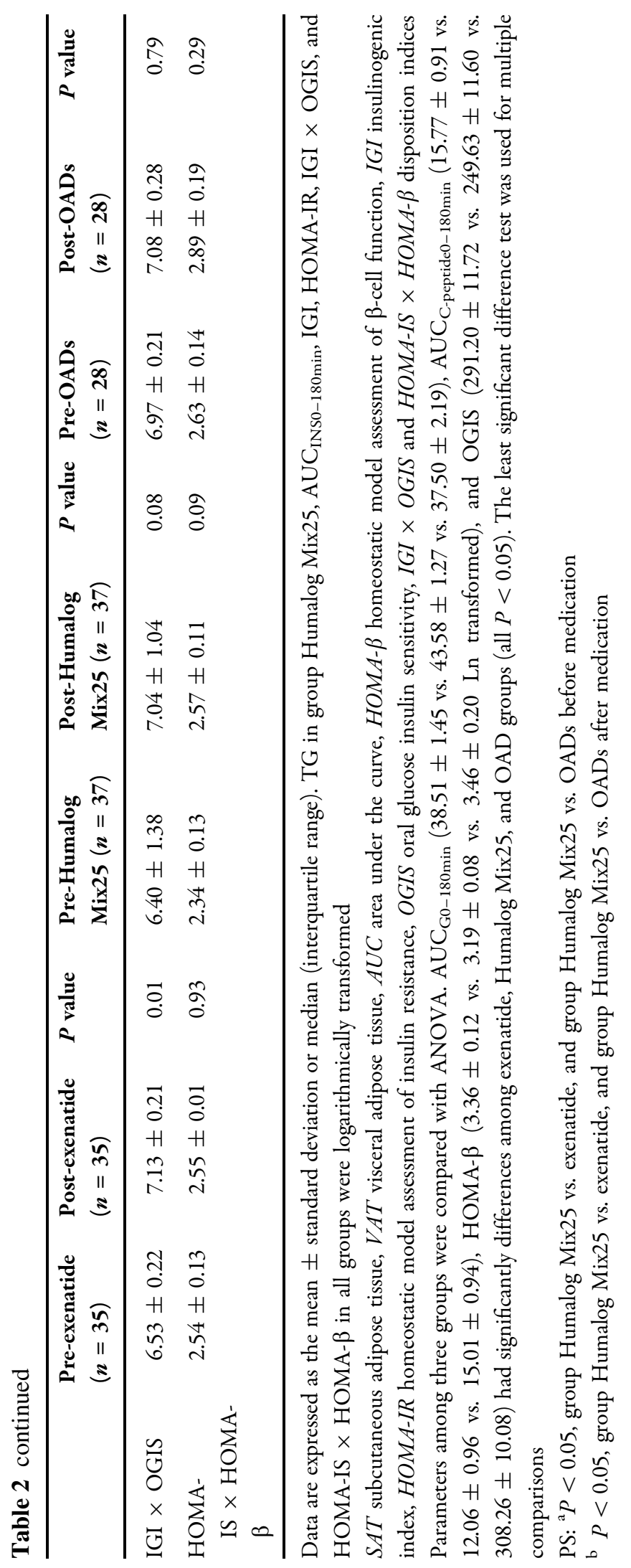



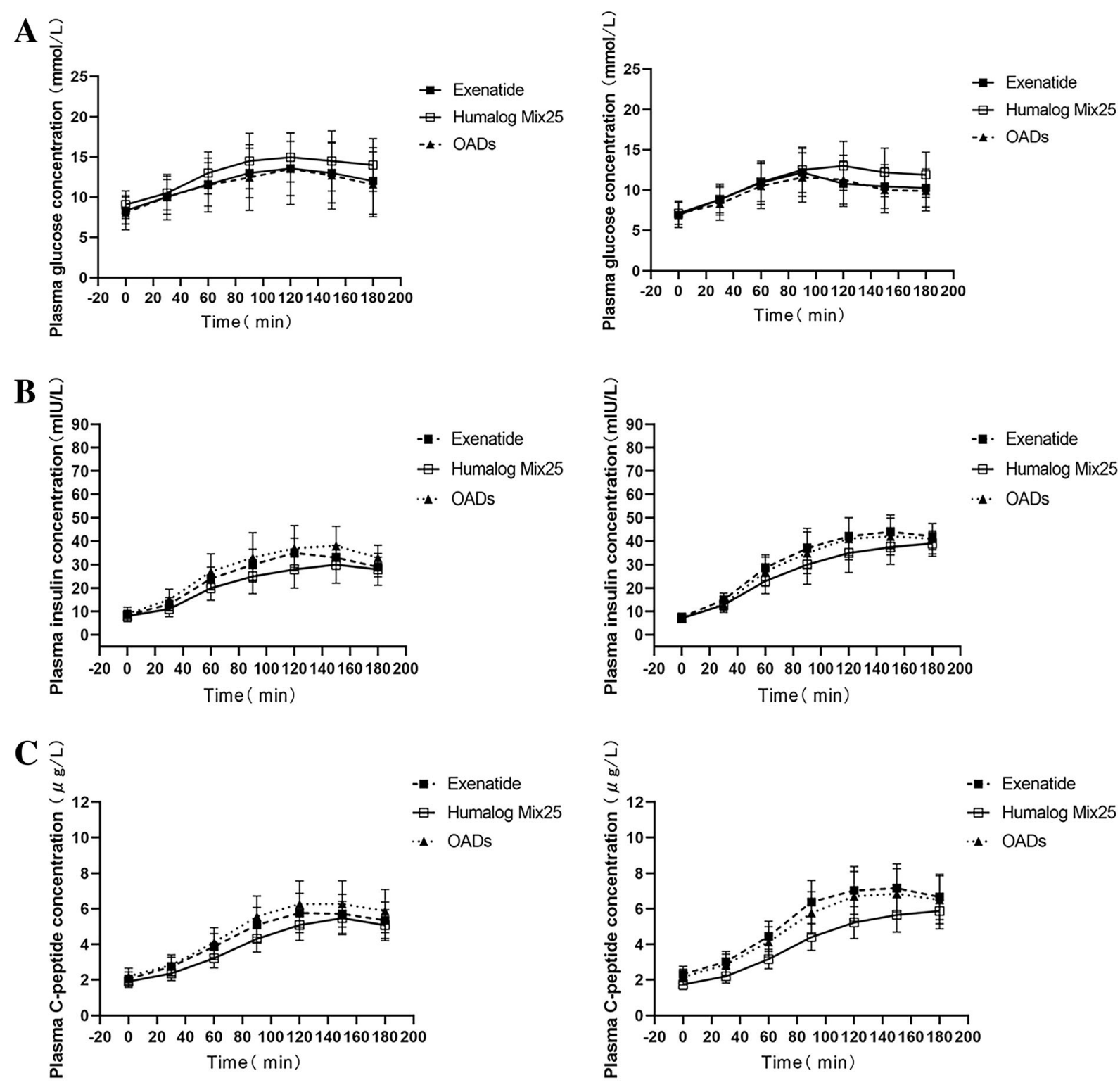

Fig. 1 Blood glucose (a), insulin (b), and c-peptide (c) response profiles on oral glucose tolerance tests before (on the left) and after (on the right) medication.

\section{Insulin Sensitivity, Insulin Resistance, Insulin Secretion, and Disposition Indices}

HOMA-IR indices were not significantly altered after 6 months of treatment in all groups. IGI significantly improved in the exenatide group $(P=0.01)$ but not in the Humalog Mix25 $(P=0.08)$ and OAD $(P=0.97)$ groups. OGIS increased after OADs therapy $(P=0.04)$

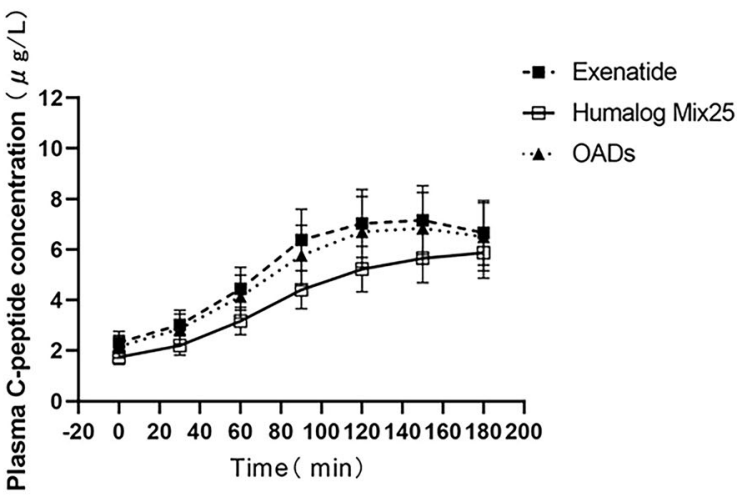

(Exenatide group, black squares; Humalog Mix25 group, white squares; OAD group, black triangles)

compared to that after exenatide $(P=0.30)$ and Humalog Mix25 treatments $(P=0.78)$. After treatment, compared with the other groups, HOMA- $\beta$ and OGIS were lower in the Humalog Mix25 group (all $P<0.05$ ) (Table 2 ). IGI $\times$ OGIS was higher after exenatide therapy $(P=0.01)$, but there were no significant differences in the other two groups. No significant 
differences in HOMA-IS $\times$ HOMA- $\beta$ were observed in all three groups (Table 2 ).

\section{Glucose Peak Time Shifts}

Before treatment, most patients reached peak glucose levels at $120 \mathrm{~min}$ (50\% of the Humalog Mix25 group, $34.8 \%$ of the OADs group, and $29.6 \%$ of the exenatide group) (Fig. 2a). After treatment, peak glucose levels shifted to 60 or 90 min in more than half of the patients in the exenatide group. No significant change was noted in the OAD and Humalog Mix25 groups (Fig. 2b).

A comparison of the three groups revealed that $57.1 \%, 27.0 \%$, and $35.7 \%$ of the patients in the exenatide, Humalog Mix25, and OAD groups shifted to an earlier peak, respectively, and this difference was statistically significant $(P=0.029)$ (Fig. 3).
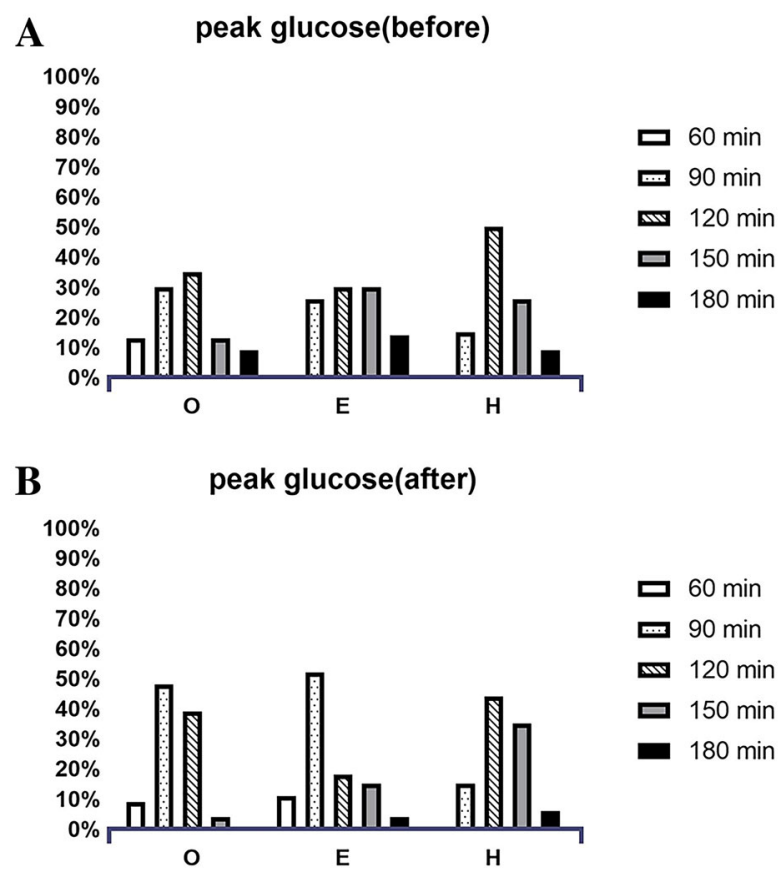

Fig. 2 Proportion of participants in each treatment with peak glucose at $60 \mathrm{~min}$ (white bars), $90 \mathrm{~min}$ (stippled bars), $120 \mathrm{~min}$ (hatched bars), $150 \mathrm{~min}$ (gray bars), and $180 \mathrm{~min}$ (black bars) before (a) and after (b) medication. $P$ values for the comparison among three groups at each visit are as follows: before, $P=0.092$; after, $P=0.005$. E exenatide, H Humalog Mix25, O OADs

\section{Multinominal Logistic Regression}

For multinominal logistic regression, the occurrence and direction of shifts in glucose peak time were considered as unordered categorical dependent variables. Independent variables included age, diabetes duration, BMI, SBP, DBP, waistline circumference, HbA1c, IGI, HOMA-IR, HOMA- $\beta$, OGIS, and IGI $\times$ OGIS. OGIS (odds ratio [OR] 0.54, 95\% confidence interval [CI] $0.33-0.89, P=0.026)$ and IGI $\times$ OGIS (OR 1.72, 95\% CI 0.44-6.68, $P=0.012$ ) were independently related to shifts in glucose peak time.

\section{DISCUSSION}

This study directly compared the effects of three different hypoglycemic drugs on $\beta$-cell function. The deficiency of $\beta$-cell function underpins elevated blood glucose levels in patients with T2DM. Moreover, progressive $\beta$-cell failure underscores the difficulty in blood glucose control in later stages of T2DM [18]. Therefore, it is important for patients with T2DM to prevent and repair damaged islet function.

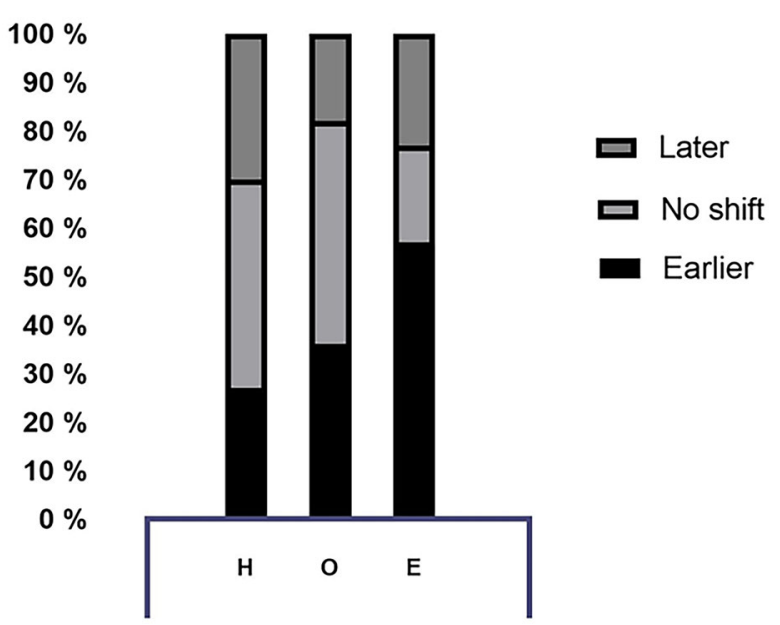

Fig. 3 Proportion of participants in the exenitide $(n=35)$, Humalog Mix25 $(n=37)$, and OAD $(n=28)$ groups that had the following shifts in timing of peak glucose before and after medication: shift to an earlier peak (black bars), no shift (light gray bars), or shift to a later peak (dark gray bars). E exenatide, $\mathrm{H}$ Humalog Mix25, O OADs 
Exenatide, insulin, metformin, acarbose, and sulfonylureas exert $\beta$-cell-protective effects [13-15], which is consistent with our results. Nonetheless, because of the lack of comparison, it remains unknown whether these effects vary between treatments.

To investigate pancreatic secretory function, we evaluated HOMA- $\beta$ and IGI. Meanwhile, insulin sensitivity was clarified by HOMA-IR and OGIS. The exenatide and OAD groups exhibited superior HOMA- $\beta$ and OGIS when compared with the Humalog Mix25 group, suggesting that exenatide and OAD treatment have greater benefits for improving both $\beta$-cell function and insulin sensitivity. HOMA- $\beta$ is an index of fasting blood glucose to insulin concentrations and is strongly related to hepatic insulin secretion. OGIS is an index of insulin sensitivity related to peripheral insulin resistance. Metformin effectively reduces blood glucose by inhibiting liver glycogen output and promoting glucose utilization by peripheral tissues. It improves insulin dysfunction by positively regulating the GLP-1 receptor expression in islet $\beta$-cells [19]. Sulfonylureas primarily act on isolated $\beta$-cells, causing increased insulin secretion to improve pancreatic $\beta$-cell function [20]; however, any effects on the peripheral insulin sensitivity are either slight or secondary to the improvement of the secretory capacity of the pancreatic islets [21]. Alpha-glucosidase inhibitors do not directly affect insulin secretion or sensitivity. They act on carbohydrate metabolism to increase L-cell activity, thereby promoting incretin secretion and indirectly improving insulin secretion or sensitivity in patients with T2DM by reducing glucose toxicity [22]. Patients treated with exenatide exhibited the greatest improvement in $\beta$-cell function because of the binding of the drug to $\beta$-cell GLP1 receptors, which enhances glucose-induced insulin synthesis and secretion [23]. GLP-1 protects $\beta$-cells from apoptosis by increasing the activity of the autocrine loop of the IGF-II/IGF-I receptor [24]. In summary, exenatide not only increases $\beta$-cell mass but also reduces apoptosis. Meanwhile, in the LIBRA trial, the improvement in $\beta$-cell secretion capacity may be due to weight loss and/or lower blood glucose levels rather than the direct effect of GLP-1 receptor agonist [25].

The glucose peak time in OGTT is of interest to researchers owing to its abilities to improve $\beta$ cell function and glucose metabolism over a period of time [11]. To the best of our knowledge, this study is the first to present a direct comparison of the effects of different antidiabetic drugs on glucose disposal during an OGTT. We observed that peak glucose time shifted forward in patients receiving exenatide, which was associated with increased $\beta$-cell function. In other words, the blood glucose peak of the exenatide group shifted forward; even if it was the effect of the drug itself, it also suggested that the drug improved the function of pancreatic $\beta$-cells. Our study participants were diagnosed with diabetes between 7 and 9 years ago, which meant that exenatide may improve pancreatic $\beta$-cell exacerbation in patients with long-term diabetes. In this study, the AUC of C-peptide and insulin decreased but that of HbA1c and 2hPBG increased with delayed glucose peak time; this indicated that the function of pancreatic islet $\beta$-cells decreased while the glycemic variability gradually increased, consistent with previous findings [10]. Inflammation, oxidative stress, and endothelial dysfunction caused by glycemic variability can lead to the occurrence of longterm diabetes complications $[26,27]$. In the future, we will further study the correlation between changes in glucose peak time and complications of long-term diabetes.

Through the construction of a regression model, we observed that OGIS and IGI $\times$ OGIS were independently related to the shifts in glucose peak time. OGIS was correlated with glucose clamp and was more accurate than other insulin sensitivity indices such as HOMAIR [28]. Additionally, it was associated with peripheral insulin sensitivity, such as sensitivity in muscle or adipose tissues [29]. IGI is used to assess the function of first-phase insulin secretion [30]. Some findings indicated that IGI may be one of the key factors in the progression from normal glucose tolerance to T2DM, leading to postprandial hyperglycemia and preventing diabetes complications [31, 32]. Because of the increase in insulin resistance in diabetes, insulin 
secretion may be enhanced as a compensatory effect; however, this does not indicate normal islet function. Accordingly, some studies have introduced the disposition/ $\beta$-cell function index adjusted using an insulin resistance index [33]. The disposition index emphasized that $\beta$ cell function was of great significance for T2DM progression [34]. Therefore, peripheral insulin sensitivity and early-phase insulin secretion contributed to changes in glucose peak time in patients with T2DM.

This study demonstrated that all three antidiabetic treatment options reduced HbA1c in patients with T2DM. The UK Prospective Diabetes Study revealed that for every $1 \%$ reduction in $\mathrm{HbA1c}$, the risks of death, myocardial infarction, or microvascular complications due to diabetes are reduced by $21 \%$, $14 \%$, or $37 \%$, respectively [35]. Furthermore, all three antidiabetic drugs resulted in decreased FBG and 2-h PBG. In particular, FBG and 2-h PBG levels of patients in the exenatide group reached the target levels of $<7.0 \mathrm{mmol} / \mathrm{L}$ and $<11.1 \mathrm{mmol} / \mathrm{L}$, respectively. The Diabetes Control and Complications Trial group speculated that factors other than HbA1c, such as the degree of postprandial glycemic fluctuation, should be considered when evaluating glycemic control and the possibility of chronic diabetic complications [36]. Exenatide decreases PBG by markedly suppressing duodenal motility and flow, slowing small intestinal transit, and decreasing 3-OMG absorption [37]. In the case of diabetes, many large-scale prospective epidemiological studies have demonstrated that postprandial hyperglycemia increases the risk of cardiovascular disease [38]. Thus, improving postprandial hyperglycemia is particularly important for the better health status of patients with diabetes.

Body weight, BMI, and waistline circumference of exenatide and OAD groups were significantly decreased after treatment, with greatest effects noted in the exenatide group. GLP-1RA treatment-related weight loss was associated with delayed gastric emptying and central appetite suppression [38]. Sulfonylureas increase body weight because they promote insulin secretion, while insulin inhibits lipolysis and promotes energy storage [14]. However, in this study, the body weight of patients in the OAD group was decreased. This may be related to the subjects' diet and exercise regimens. Meanwhile, metformin exerted a weight loss effect [39]. Furthermore, obesity can cause insulin sensitivity disorders through mechanisms such as inflammatory factors, immune cytokines, and so on [40]. In this study, the exenatide and $\mathrm{OAD}$ groups have improved insulin sensitivity after weight control. Nevertheless, only exenatide improved ectopic fat accumulation. In vivo and in vitro experiments and a series of mechanism studies have proved that exenatide reduces the acetylation level of heat shock factor 1 through deacetylase SIRT1; this upregulates heat shock protein expression and improves liver endoplasmic reticulum stress and lipid deposition caused by lipotoxicity [41]. Notably, VAT and SAT can serve as markers of T2DM and cardiometabolic risk [42, 43]. In summary, exenatide therapy is beneficial for controlling weight and reducing the risk of cardiovascular events by improving ectopic fat deposition.

This study has limitations owing to its insufficient sample size and investigation time. In particular, improvements of $\beta$-cell function required long-term evaluation of treatment methods to determine whether the beneficial effects continued and delayed disease progression. In the follow-up study, we plan to further expand the sample size and analyze the glucose peak time for a longer period as an evaluation of the effect of hypoglycemic drugs on the $\beta$-cell function of patients with diabetes. Second, shifts in glucose peak time may be affected by gastric emptying or carbohydrate load before the OGTT test. In the future, further studies are required to elucidate the effects of serum glucagon and glucose-dependent insulinotropic polypeptide on glucose peak time. Third, there are many kinds of oral hypoglycemic drugs, and follow-up studies should further compare the effects of different oral drugs on the time of peak blood glucose. Fourth, it is better to use a meal tolerance test (MTT) instead of OGTT because of the burden of risk of unnecessary hyperglycemia in patients with T2DM. We will further optimize the plan in follow-up research. 


\section{CONCLUSIONS}

Exenatide, Humalog Mix25, and OADs may improve glycemic metabolism. Exenatide improves insulin secretion, insulin sensitivity, and fat deposition. Furthermore, glucose peak time is effective in assessing islet $\beta$-cell function in patients with T2DM. This study highlights a novel indicator that is simple and effective for evaluating the efficacy of hypoglycemic drugs in patients with T2DM.

\section{ACKNOWLEDGEMENTS}

We thank the participants, study nurses, and Jingyi Zhu for her assistance in laboratory technology.

Funding. This research was supported by the grants from Nantong Municipal Science and Technology Project (grant numbers JC2020046, JC2020047, MS22019005, MA2020024, JC20 19121), Jiangsu Provincial Six Talent Peaks for high-level talents (grant numbers 2016-WSN098), Chinese Association of Integrated Traditional and Western Medicine - Scientific Research Fund of He-Huang Pharmaceutical (Grant numbers HMP2008007 ${ }^{\mathrm{P}}$ ). The journal's Rapid Service Fee was funded by the authors.

Editorial Assistance. We thank Editage (www.editage.cn) for English language editing (funded by the authors).

Authorship. All named authors meet the International Committee of Medical Journal Editors (ICMJE) criteria for authorship for this article, take responsibility for the integrity of the work as a whole, and have given their approval for this version to be published.

Authors' Contributions. Yunjuan Gu, Xinlei Wang, Shiwei Cui and Yanqiu Jiang have made substantial contributions to the conception of the work, the design of the work, the interpretation of data and the drafting of the manuscript. Rongping Zhang, Xiaoqin Zhao, Lili Yao, Rong OuYang, Wei Chen, Ranran Zhou and Xuying Zhao have made substantial contributions to the acquisition of data and the analysis of data. Zhuqi Tang, Jin Yuan, Jie Yuan, Chen Qian and Ping Huang have made substantial contributions to the assessment of results and the interpretation of data.

Disclosures. Yanqiu Jiang, Shiwei Cui, Rongping Zhang, Xiaoqin Zhao, Lili Yao, Rong OuYang, Wei Chen, Ranran Zhou, Xuying Zhao, Zhuqi Tang, Jin Yuan, Jie Yuan, Chen Qian, Ping Huang, Yunjuan $\mathrm{Gu}$ and Xinlei Wang declare no potential conflict of interest about this article.

Compliance with Ethics Guidelines. All participants signed an informed consent form in the study. The protocol was approved by the Ethics Committee of the Affiliated Hospital of Nantong University (approval number 2015-K002-D01). The study was registered with Chinese Clinical Trial Registry (ChiCTR-IPR14005568). The study was carried out in line with the Helsinki Declaration of 1964 and its later amendments.

Data Availability. The datasets used to support the findings of this study are available from the corresponding author upon request.

Open Access. This article is licensed under a Creative Commons Attribution-NonCommercial 4.0 International License, which permits any non-commercial use, sharing, adaptation, distribution and reproduction in any medium or format, as long as you give appropriate credit to the original author(s) and the source, provide a link to the Creative Commons licence, and indicate if changes were made. The images or other third party material in this article are included in the article's Creative Commons licence, unless indicated otherwise in a credit line to the material. If material is not included in the article's Creative Commons licence and your intended use is not permitted by statutory regulation or exceeds the permitted use, you will need to obtain permission directly from the copyright holder. To view a copy of this licence, visit http://creativecommons.org/licenses/by$\mathrm{nc} / 4.0 /$. 


\section{REFERENCES}

1. DeFronzo RA, Tobin JD, Andres R. Glucose clamp technique: a method for quantifying insulin secretion and resistance. Am J Physiol. 1979;237(3):E214-23.

2. Chen ME, Aguirre RS, Hannon TS. Methods for measuring risk for type 2 diabetes in youth: the oral glucose tolerance test (OGTT). Curr Diab Rep. 2018;18(8):51.

3. Leonetti F, Iacobellis G, Zappaterreno A, et al. Insulin sensitivity assessment in uncomplicated obese women: comparison of indices from fasting and oral glucose load with euglycemic hyperinsulinemic clamp. Nutr Metab Cardiovasc Dis. 2004;14(6):366-72.

4. Monzillo LU, Hamdy O. Evaluation of insulin sensitivity in clinical practice and in research settings. Nutr Rev. 2003;61(12):397-412.

5. Mari A, Pacini G, Murphy E, Ludvik B, Nolan JJ. A model-based method for assessing insulin sensitivity from the oral glucose tolerance test. Diabetes Care. 2001;24(3):539-48.

6. Piché M-E, Lemieux S, Corneau L, Nadeau A, Bergeron J, Weisnagel SJ. Measuring insulin sensitivity in postmenopausal women covering a range of glucose tolerance: comparison of indices derived from the oral glucose tolerance test with the euglycemic-hyperinsulinemic clamp. Metabolism. 2007;56(9):1159-66.

7. Kanauchi M, Kimura K, Kanauchi K, Saito Y. Betacell function and insulin sensitivity contribute to the shape of plasma glucose curve during an oral glucose tolerance test in non-diabetic individuals. Int J Clin Pract. 2005;59(4):427-32.

8. Tura A, Morbiducci U, Sbrignadello S, Winhofer Y, Pacini G, Kautzky-Willer A. Shape of glucose, insulin, C-peptide curves during a 3-h oral glucose tolerance test: any relationship with the degree of glucose tolerance? Am J Physiol Regul Integr Comp Physiol. 2011;300(4):R941-8.

9. Manco M, Nolfe G, Pataky Z, Winhofer Y, Pacini G, Kautzky-Willer A. Shape of the OGTT glucose curve and risk of impaired glucose metabolism in the EGIR-RISC cohort. Metabolism. 2017;70:42-50.

10. Wang X, Zhao X, Zhou R, et al. Delay in glucose peak time during the oral glucose tolerance test as an indicator of insulin resistance and insulin secretion in type 2 diabetes patients. J Diabetes Investig. 2018;9(6):1288-95.

11. Kramer CK, Vuksan V, Choi H, Zinman B, Retnakaran R. Emerging parameters of the insulin and glucose response on the oral glucose tolerance test: reproducibility and implications for glucose homeostasis in individuals with and without diabetes. Diabetes Res Clin Pract. 2014;105(1):88-95.

12. Tran S, Kramer CK, Zinman B, Choi H, Retnakaran R. Effect of chronic liraglutide therapy and its withdrawal on time to postchallenge peak glucose in type 2 diabetes. Am J Physiol Endocrinol Metab. 2018;314(3):E287-95.

13. Gastaldelli A, Brodows RG, D'Alessio D. The effect of chronic twice daily exenatide treatment on $\beta$-cell function in new onset type 2 diabetes. Clin Endocrinol (Oxf). 2014;80(4):545-53.

14. Hsu PF, Sung SH, Cheng HM, et al. Cardiovascular benefits of acarbose vs sulfonylureas in patients with type 2 diabetes treated with metformin. J Clin Endocrinol Metab. 2018;103(10):3611-9.

15. Koledova RE, Metcalfe S, Hultman C, Milicevic Z, Romania/Russia Mix25 Study Group. Glycemic control with humalog Mix25 in type 2 diabetes inadequately controlled with glyburide. Clin Ther. 2001;23(10):1732-44.

16. Wallace TM, Levy JC, Matthews DR. Use and abuse of HOMA modelling. Diabetes Care. 2004;27(6): 1487-95.

17. Cortez-Navarrete M, Martínez-Abundis E, PérezRubio KG, González-Ortiz M, Villar MM-D. Momordica charantia administration improves insulin secretion in type 2 diabetes mellitus. J Med Food. 2018;21(7):672-7.

18. Ohn JH, Kwak SH, Cho YM, et al. 10-year trajectory of $\beta$-cell function and insulin sensitivity in the development of type 2 diabetes: a communitybased prospective cohort study. Lancet Diabetes Endocrinol. 2016;4(1):27-34.

19. Liu Y, Hong T. Combination therapy of dipeptidyl peptidase- 4 inhibitors and melformin in type 2 diabetes: rationale and evidence. Diabetes Obes Metab. 2014;16(2):111-7.

20. Kolterman OG, Gray RS, Shapiro G, Scarlett JA, Griffin J, Olefsky JM. The acute and chronic effects of sulfonylurea therapy in type II diabetic subjects. Diabetes. 1984;33(4):346-54.

21. Matthews DR, Hosker JP, Stratton I. The physiological action of gliclazide: beta-cell function and insulin resistance. Diabetes Res Clin Pract. 1991;14(Suppl 2):S53-9.

22. Delgado H, Lehmann T, Bobbioni-Harsch E, Ybarra J, Golay A. Acarbose improves indirectly both insulin resistance and secretion in obese type 2 diabetic patients. Diabetes Metab. 2002;28(3):195-200. 
23. Drucker DJ. The biology of incretin hormones. Cell Metab. 2006;3(3):153-65.

24. Cornu M, Yang J-Y, Jaccard E, Poussin C, Widmann C, Thorens B. Glucagon-like peptide-1 protects beta-cells against apoptosis by increasing the activity of an IGF-2/IGF-1 receptor autocrine loop. Diabetes. 2009;58(8):1816-25.

25. Retnakaran R, Kramer CK, Choi H, et al. Liraglutide and the preservation of pancreatic b-cell function in early type 2 diabetes: the LIBRA Trial. Diabetes Care. 2014;37(12):3270-8.

26. Monnier L, Colette C. Postprandial and basal hyperglycaemia in type 2 diabetes: contributions to overall glucose exposure and diabetic complications. Diabetes Metab. 2015;41(6):6S9-6S15.

27. Diabetes Prevention Program Research Group. Long-term effects of lifestyle intervention or metformin on diabetes development and microvascular complications over 15-year follow-up: the diabetes prevention program outcomes study. Lancet Diabetes Endocrinol. 2015;3(11):866-75.

28. Thomaseth K, Pavan A, Berria R, Glass L, DeFronzo $\mathrm{R}$, Gastaldelli A. Model-based assessment of insulin sensitivity of glucose disposal and endogenous glucose production from double-tracer oral glucose tolerance test. Comput Methods Programs Biomed. 2008;89(2):132-40.

29. Hsieh C-H, Chung-Ze Wu, Hsiao F-C, et al. The impact of metabolic syndrome on insulin sensitivity, glucose sensitivity, and acute insulin response after glucose load in early-onset type 2 diabetes mellitus: Taiwan Early-Onset Type 2 Diabetes Cohort Study. Metabolism. 2008;57(11):1615-21.

30. Rudenski AS, Hadden DR, Atkinson AB, et al. The interleukin-1 receptor antagonist anakinra improves first-phase insulin secretion and insulinogenic index in subjects with impaired glucose tolerance. Diabetes Obes Metab. 2014;16(12): 1269-73.

31. Kondo Y, Harada N, Hamasaki A, et al. Sitagliptin monotherapy has better effect on insulinogenic index than glimepiride monotherapy in Japanese patients with type 2 diabetes mellitus: a 52 -week, multicenter, parallel-group randomized controlled trial. Diabetol Metab Syndr. 2016;8:15.

32. Mulvey CK, McNeill AM, Girman CJ, et al. Differential associations of oral glucose tolerance testderived measures of insulin sensitivity and pancreatic beta-cell function with coronary artery calcification and microalbuminuria in type 2 diabetes. Diabetes Care. 2014;37:124-33.
33. Lyssenko V, Almgren P, Anevski D, et al. Predictors of and longitudinal changes in insulin sensitivity and secretion preceding onset of type 2 diabetes. Diabetes. 2005;54(1):166-74.

34. Utzschneider KM, Prigeon RL, Faulenbach MV, et al. Oral disposition index predicts the development of future diabetes above and beyond fasting and 2-h glucose levels. Diabetes Care. 2009;32(2): 335-41.

35. Stratton IM, Adler AI, Neil HA, et al. Association of glycaemia with macrovascular and microvascular complications of type 2 diabetes (UKPDS 35): prospective observational study. $\mathrm{Br}$ Med J. 2000;321(7258):405-12.

36. Diabetes Control and Complications Trial Research Group. The relationship of glycemic exposure (HbA1c) to the risk of development and progression of retinopathy in the Diabetes Control and Complications Trial. Diabetes. 1995;44(8):968-83.

37. Thazhath SS, Marathe CS, Wu T, et al. The glucagon-like peptide 1 receptor agonist exenatide inhibits small intestinal motility, flow, transit, and absorption of glucose in healthy subjects and patients with type 2 diabetes: a randomized controlled trial. Diabetes. 2016;65(1):269-75.

38. Kanoski SE, Fortin SM, Arnold M, Grill JJ, Hayes MR. Peripheral and central GLP-1 receptor populations mediate the anorectic effects of peripherally administered GLP-1 receptor agonists, liraglutide and exendin-4. Endocrinology. 2011;152(8): 3103-12.

39. Liu C-Y, Chang T-C, Lin S-H, Sheng-Tang Wu, Cha T-L, Tsao C-W. Metformin ameliorates testicular function and spermatogenesis in male mice with high-fat and high-cholesterol diet-induced obesity. Nutrients. 2020;12(7):1932.

40. Boßlau TK, Wasserfurth P, Krüger B, et al. Abdominal obesity-related disturbance of insulin sensitivity is associated with CD8+ EMRA cells in the elderly. Cells. 2021;10(5):998.

41. Zheng $\mathrm{X}$, Fen $\mathrm{Xu}$, Liang $\mathrm{H}$, et al. SIRT1/HSF1/HSP pathway is essential for exenatide-alleviated, lipidinduced hepatic endoplasmic reticulum stress. Hepatology. 2017;66(3):809-24.

42. van der Meer RW, Lamb HJ, Smit JW, de Roos A. MR imaging evaluation of cardiovascular risk in metabolic syndrome. Radiology. 2012;264(1):21-37.

43. Kouidhi S, Berrhouma R, Rouissi K, et al. Human subcutaneous adipose tissue Glut 4 mRNA expression in obesity and type 2 diabetes. Acta Diabetol. 2013;50(2):227-32. 